\title{
NONDENTABLE SOLID SUBSETS IN BANACH LATTICES FAILING RNP. APPLICATIONS TO RENORMINGS
}

\author{
ELISABETH WERNER
}

(Communicated by William J. Davis)

\begin{abstract}
We show that for every Banach lattice $E$ failing RNP and not containing $c_{0}$ (resp. containing $c_{0}$ ) and for every $\varepsilon>0$ there exists a solid convex closed subset $D$ of the unit ball of $E$, such that

$$
\operatorname{dist}\left[\operatorname{ext}\left(\bar{D}^{\sigma\left(E^{* *}, E^{*}\right)}\right), E\right]>1-\varepsilon\left(\text { resp. }>\frac{1}{2}-\varepsilon\right)
$$

and such that every slice of $D$ has diameter bigger than $2-\varepsilon$.

We also prove that these results are optimal. We apply them to construct rough lattice norms with almost optimal constant on non-Asplund Banach lattices.
\end{abstract}

\section{INTRODUCTION}

In [9] extremal behaviour of nondentability in Banach spaces failing the Radon-Nikodym property (RNP) was studied from two points of view. The first one deals with the existence of convex sets with only "big slices", the other one deals with a characterization of non-RNP spaces due to Bourgain [1] and Stegall [11]: the existence of a convex set $C$ such that the extreme points of the $w^{*}$-closure of $C$ in the bidual are "far" from the space.

In the first section of this paper we investigate the same phenomena for Banach lattices failing RNP, restricting ourselves to solid convex sets (thus taking into account the lattice structure).

The results we obtain are the best possible. Furthermore, no separability assumption is needed, in contrast with the results obtained in [9]. It turns out that there is a big difference for both the obtained results and the methods of the proofs, according to whether the Banach lattice contains $c_{0}$ or not. The precise statements are described in Theorem 1 and Theorem 2. Note that these two theorems are valid for every lattice norm.

In the second section we show that there exist equivalent lattice norms for which the "bad" convex solid sets are the unit balls. Moreover, the constants are the best possible (see Theorem 11 for the precise statement).

Received by the editors September 29, 1987 and, in revised form, January 20, 1987.

1980 Mathematics Subject Classification (1985 Revision). Primary 46B05, 46G10. 
Similar methods are used to prove that every non-Asplund Banach lattice has an equivalent lattice norm which is uniformly non-Fréchet-differentiable, with almost optimal constant.

Notations. $X, Y$ will always denote Banach spaces, $E, F$ will denote Banach lattices. The unit balls will be denoted $B(X)$ resp. $B(E)$.

We write $E_{+}$for the positive cone of a Banach lattice $E$. If $E$ is a band in its bidual $E^{* *}$, we denote by $E_{S}$ the band orthogonal to $E$ in $E^{* *}$.

For a convex subset $C$ of $X, \operatorname{ext}(C)$ is the set of extreme points of $C ; \widetilde{C}$ is the $\omega^{*}$-closure of $C$ in $X^{* *}$. A (open) slice of $C$ is a subset $S$ of $C$ of the form $S\left(C, x^{*}, \alpha\right)=\left\{x \in C:\left\langle x^{*}, x\right\rangle>M_{x^{*}}-\alpha\right\}$, where $x^{*} \in X^{*},\left\|x^{*}\right\|=1$, $\alpha>0$, and $M_{x^{*}}=\sup _{x \in C}\left\langle x^{*}, x\right\rangle$.

For a subset $C$ of $E, C_{+}=E_{+} \cap C$ is the positive part of $C ; S(C)=\{x \in$ $E: \exists c \in C$ such that $|x| \leq|c|\}$ is the solid hull of $C$.

For $x, y \in E$ we denote by $[x, y]_{E}=[x, y]=\{z \in E: x \leq z \leq y\}$ the order interval in $E$ defined by $x$ and $y$.

For $x \in E$, we write $I(x)$ for the ideal in $E$ generated by $x$. It is well known (see [10]), that there exists a compact space $K_{x}$ and an isometric lattice isomorphism $i_{x}$ between $I(x)$ and $C\left(K_{x}\right)$ such that $\mathbf{1}_{K_{x}}=i_{x}(|x|)$. For unexplained notation concerning Banach lattices (resp. RNP) we refer to [10] (resp. [4]).

\section{The MAIN RESUltS}

Theorem 1. Let $E$ be a Banach lattice containing $c_{0}$. Then for every $\varepsilon>0$ there exists a solid closed convex subset $D$ of $B(E)$ such that

(i) $\operatorname{dist}[\operatorname{ext}(\widetilde{D}), E]>\frac{1}{2}-\varepsilon$

(ii) for every slice $S$ of $D$ one has: $\operatorname{diam} S>2-\varepsilon$.

Theorem 2. Let $E$ be a Banach lattice not containing $c_{0}$ and failing RNP. Then for every $\varepsilon>0$ there exists a solid, closed, convex subset $D$ of $B(E)$ such that

(i) $\operatorname{ext}(\widetilde{D}) \subseteq E_{s}$ and $\operatorname{dist}[\operatorname{ext}(\widetilde{D}), E]>1-\varepsilon$

(ii) for every slice $S$ of $D$ one has: $\operatorname{diam} S>2-\varepsilon$.

Remark 3. (i) We will show in Proposition 8 that the value $\frac{1}{2}-\varepsilon$ in Theorem 1 ,(i) is optimal, even for separable Banach lattices. However, in the separable case, the value $\frac{1}{2}-\varepsilon$ can be replaced by $1-\varepsilon$, if $C$ is allowed to be nonsolid [9].

(ii) Notice that no separability assumption is needed in Theorem 2,(i). (Compare with [9].)

(iii) It is clear that the value 2 in Theorem 1,(ii) and Theorem 2,(ii) is optimal. This optimal value is not "attained" if $E$ is merely supposed to be a (even separable) Banach space [9].

We will frequently make use of the following lemmas. They are probably known, even if we were unable to find a reference for them. We include the 
proofs for completeness. The proof in the nonseparable case of Lemma 5 was pointed out to us by F. Räbiger.

Lemma 4. Let $E$ be a Banach lattice.

(i) Let $D$ be a solid, convex subset of $E$. If $e \in \operatorname{ext}(D)$, then $|e| \in \operatorname{ext}(D)$.

(ii) Let $C$ be a convex subset of $E_{+}$. If $e \in \operatorname{ext}(S(C))$, then $|e| \in \operatorname{ext}(C)$.

Proof. (i) The proof will be done in two steps. We will also make use of the following well-known fact:

For a compact space $K$ the extreme points of $B(C(K))$ are the continuous functions $\varphi$ satisfying $|\varphi| \equiv 1$.

Step 1. We are going to prove that if $e \in \operatorname{ext}(D)$ and $y \in D$ are such that $|e| \leq|y|$, then $|e|=|y|$. Indeed, since $D$ is solid, $[-|y|,|y|] \subseteq D$ and, as $e$ is an extreme point of $D, e$ is also an extreme point of $[-|y|,|y|]$. By the fact mentioned above we have: $\left|i_{|y|}(e)\right|=1 \quad\left(=\mathbf{1}_{K_{|y|}}\right)$. Hence $i_{|y|}(|e|)=\left|i_{|y|}(e)\right|=$ $1=i_{|y|}(|y|)$. Therefore $|e|=|y|$.

Step 2. Let $y_{1}, y_{2} \in D$ such that $|e|=\frac{1}{2}\left(y_{1}+y_{2}\right)$. By step 1 this implies that $|e|=\frac{1}{2}\left(\left|y_{1}\right|+\left|y_{2}\right|\right)$. In particular, $\left|y_{1}\right|,\left|y_{2}\right| \in I(|e|)$. Hence

$$
i_{|e|}(e)=i_{|e|}(e) \cdot \frac{i_{|e|}\left(y_{1}\right)+i_{|e|}\left(y_{2}\right)}{2} \text {. }
$$

Since $D$ is solid, there exist $z_{1}, z_{2} \in D$ such that $i_{|e|}\left(z_{i}\right)=i_{|e|}(e) \cdot i_{|e|}\left(y_{i}\right)$. As $e$ is an extreme point of $D$, this implies that $z_{1}=z_{2}=e$, hence by definition we have: $i_{|e|}(e)=i_{|e|}(e) \cdot i_{|e|}\left(y_{1}\right)$, which implies that $i_{|e|}\left(y_{1}\right)=1 \quad\left(=\mathbf{1}_{K_{|e|}}\right)$, since $\left|i_{|e|}(e)\right|=1$. Then $y_{1}=|e|$. Similarly $y_{2}=|e|$. This concludes the proof of (i).

(ii) If $e \in \operatorname{ext}(S(C))$, then by (i) $|e| \in \operatorname{ext}(S(C))$. Hence by definition of the solid hull there exists $y \in C$ such that $|e| \leq y$. It follows from the proof of (i) that $|e|=y$ and (ii) is proved.

Lemma 5. Let $E$ be a Banach lattice and $D$ be a convex, solid subset of $E^{*}$ (resp. E). Then $\bar{D}^{*}$ is again a convex solid subset of $E^{*}$ (resp. $\widetilde{D}$ is a convex solid subset of $E^{* *}$ ).

Proof. We are first going to show that $\bar{D}^{*}$ is again solid. Suppose this were not the case. Then there exists $x \in \bar{D}^{*}, y \in[-|x|,|x|]$ such that $y \notin \bar{D}^{*}$. By the Hahn-Banach theorem there exists $\varphi \in E$ such that

$$
\sup _{z \in D}\langle\varphi, z\rangle\langle\langle\varphi, y\rangle \leq\langle|\varphi|,|y|\rangle \leq\langle|\varphi|,|x|\rangle .
$$

As $D=\bigcup_{z \in D_{+}}[-z, z]$, we get

$$
\begin{aligned}
\sup _{z \in D}\langle\varphi, z\rangle & =\sup _{z \in D_{+}} \sup _{\left|z^{\prime}\right| \leq z}\left\langle\varphi, z^{\prime}\right\rangle \\
& =\sup _{z \in D_{+}}\langle|\varphi|, z\rangle=\sup _{z \in D}\langle|\varphi|, z\rangle \\
& =\sup _{z \in \bar{D}^{*}}\langle|\varphi|, z\rangle
\end{aligned}
$$


(see [10], page 72), and thus $\sup _{z \in \bar{D}^{*}}\langle|\varphi|, z\rangle\left\langle\langle|\varphi|,|x|\rangle\right.$. Let now $\left(x_{\gamma}\right)_{\gamma \in \Gamma} \subseteq D$ be such that $x=\omega^{*}-\lim _{\gamma} x_{\gamma}$. Define $x_{0}=\omega^{*}-\lim _{\gamma}\left|x_{\gamma}\right|$. It is clear that $x_{0} \in$ $\bar{D}^{*}$ and $x_{0} \geq|x|$ since $E_{+}^{*}$ is $\omega^{*}$-closed in $E^{*}$. Then $\langle|\varphi|,|x|\rangle \leq\left\langle|\varphi|, x_{0}\right\rangle=$ $\lim _{\gamma}\left\langle|\varphi|,\left|x_{\gamma}\right|\right\rangle$, a contradiction since $x_{0} \in \bar{D}^{*}$. This contradiction finishes the proof of the fact that $\bar{D}^{*}$ is solid.

For the second assertion notice first that for every $x \in E_{+}\left([-x, x]_{E}\right)^{\sim}=$ $[-x, x]_{E^{* *}}$. It is easily checked that $\bigcup_{x \in D_{+}}[-x, x]_{E^{* *}}$ is solid and convex. Then, by the first part,

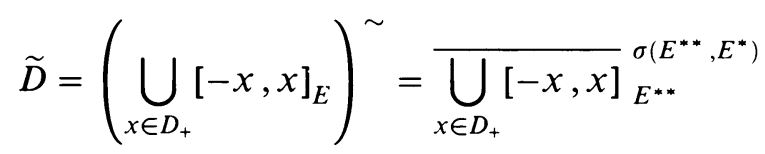

is solid.

Corollary 6. Let $E$ be a Banach lattice and let $C$ be a convex positive set in $E$ $\left(\right.$ resp. $\left.E^{*}\right)$. Then $S(\widetilde{C})=\widetilde{S(C)}\left(\right.$ resp. $\left.\overline{S(C)}^{*}=S\left(\bar{C}^{*}\right)\right)$.

Proof. From the $\omega^{*}$-closedness of $E_{+}^{* *}$ resp. $E_{+}^{*}$, it follows that $S(\widetilde{C})$ resp. $S\left(\bar{C}^{*}\right)$ is again $\omega^{*}$-closed. Then the conclusion follows from Lemma 5.

Remark. The above corollary is false if $C$ is not positive. This can be checked easily by taking $C=$ convex hull of $\left\{\delta_{1 / n}-\delta_{1 /(n+1)}: n \in \mathbf{N}\right\} \subseteq M[0,1]$.

For the proof of Theorem 1 we also need the following well-known lemma (see for example [9]).

Lemma 7. Let $X$ be a Banach space, $Y$ a subspace of $X$, and $\xi \in Y^{00}=$ $\bar{Y}^{\sigma\left(X^{* *}, X^{*}\right)}$. Then

$$
\frac{1}{2} \operatorname{dist}[\xi, Y] \leq \operatorname{dist}[\xi, X] \leq \operatorname{dist}[\xi, Y] .
$$

Proof of Theorem 1. If $E$ contains $c_{0}$, then for every $\varepsilon>0$ there exists a lattice homomorphism $R: c_{0} \rightarrow E$ such that $\|R\|=1$ and $\left\|R^{-1}\right\|<1+\varepsilon$ (use [8, II, 1.a.5] and apply James's method [8, I, 2.e.3]). We put $D=R\left(B\left(c_{0}\right)\right)$. This is clearly a solid, closed, convex subset of $B(E)$. As $\operatorname{dist}\left[\operatorname{ext}\left(\widetilde{B\left(c_{0}\right)}\right)\right.$, $\left.c_{0}\right]=1$, we deduce that $\operatorname{dist}\left[\operatorname{ext}(\widetilde{D}), R\left(c_{0}\right)\right]>1 /(1+\varepsilon)$ and (i) follows with Lemma 7.

If $S$ is a slice of $D, R^{-1}(S)$ is a slice of $B\left(c_{0}\right)$. As every slice of $B\left(c_{0}\right)$ has diameter 2 , we deduce that $S$ has diameter bigger than $2 /(1+\varepsilon)$ and (ii) follows.

The next proposition shows that the value $\frac{1}{2}$ in Theorem 1,(i) is optimal.

Proposition 8. Let $c$ be the Banach lattice of all converging sequences (over $\mathbf{N}$ ). Then for every solid, convex subset $D$ of $B(c)$ and every $e \in \operatorname{ext}(\widetilde{D})$ one has: $|e| \in \operatorname{ext}(\widetilde{D})$ and $\operatorname{dist}[|e|, c] \leq \frac{1}{2}$.

Proof. Let $D$ be a solid, convex subset of $B(c)$. By Lemma 5 we have that $\widetilde{D}$ is again solid. Let $e$ be an extreme point of $\widetilde{D}$. Lemma 4 shows that $|e|$ is also 
an extreme point of $\widetilde{D}$. As $c^{* *}=l^{\infty}(\overline{\mathbf{N}}),|e|$ is of the form $|e|=\left(\alpha_{1}, \alpha_{2}, \ldots\right)$ with $0 \leq \alpha_{i} \leq 1$ for all $i$. Hence $\||e|-x\|_{\infty} \leq \frac{1}{2}$, if we take for $x$ the sequence in $c$ such that $x=\left(\frac{1}{2}, \frac{1}{2}, \frac{1}{2}, \ldots\right)$. This implies that $\operatorname{dist}[|e|, c] \leq \frac{1}{2}$.

The proof of Theorem 2 is more complicated than the proof of Theorem 1. Besides the lemmas stated earlier, we will use completely different methods which will be developed in the sequel.

The main step in the proof of Theorem 2 is the following Theorem 9. $\mathrm{m}$ denotes the Lebesgue measure on $[0,1]$, and for $A \subseteq[0,1], m(A)>0, \mathscr{F}_{A}=$ $\left\{f \in L^{1}[0,1]: f \geq 0,\|f\|=\left\|f \cdot \chi_{A}\right\|=1\right\}[11]$.

Theorem 9. Let $E$ be a Banach lattice failing RNP and not containing $c_{0}$. Then there exists a positive nonrepresentable operator $T: L^{1}[0,1] \rightarrow E$ such that every measurable subset $A \subseteq[0,1], m(A)>0$, satisfies: $\operatorname{ext}\left(T\left(\mathscr{F}_{A}\right)\right)^{\sim} \subseteq E_{S}$.

For the proof of this theorem we will need the following result of BourgainTalagrand[2].

Theorem. Let $E$ be as in Theorem 1 and suppose also that $E$ is separable. Then there exist a positive bounded convex subset $C$ of $E_{+}$, a finitely $C$-valued martingale $\left(Y_{n}, \mathscr{A}_{n}\right)_{n \in \mathbf{N}}$ on $[0,1], \mathscr{A}_{n}$-measurable functions $f_{n}:[0,1] \rightarrow S\left(E^{*}\right)$ and $\alpha_{n}:[0,1] \rightarrow \mathbf{R}_{+} \backslash\{0\}$ such that the following properties hold:

(i) $Y_{n}(t) \in S\left(C, f_{n}(t), \alpha_{n}(t)\right)$ for every $n \in \mathbf{N}, t \in[0,1]$

(ii) $S\left(C, f_{n+1}(t), \alpha_{n+1}(t)\right) \subseteq S\left(C, f_{n}(t), \alpha_{n}(t)\right)$.

(iii) $H_{n} \cap \bar{S}\left(C, f_{n}(t), \alpha_{n}(t)\right)=\varnothing$, where $H_{n}=\left\{x \in E_{+}:\|x \wedge u\| \geq \frac{1}{n}\right\}$ and $u$ is a quasi-interior point of $E$.

Notice that we can always suppose that the complete $\sigma$-field $\tau\left(\bigcup_{n} \mathscr{A}_{n}\right)$ generated by $\bigcup_{n} \mathscr{A}_{n}$ is the Borel $\sigma$-field on $[0,1]$.

We are going to show that the operator occurring in Theorem 9 is nothing else but the canonical operator associated to the martingale given in [2] (i.e. $\left.T\left(\chi_{A}\right)=\lim _{n} \int_{A} Y_{n}(t) d m\right)$. To study the sets $T\left(\mathscr{F}_{A}\right)$ we need to "restrict" the martingale $\left(Y_{n}, \mathscr{A}_{n}\right)_{n \in \mathbf{N}}$ of [2] to $A$. More precisely we use the following notation. For every measurable subset $A$ of $[0,1], m(A)>0$, let

- $\mathscr{A}_{n}^{A}=\left.\mathscr{A}_{n}\right|_{A}$, the restricted $\sigma$-field on $A$.

- $I_{n, i}^{A}=I_{n, i} \cap A$, where $I_{n, i}(1 \leq i \leq p(n))$ are the atoms of $\mathscr{A}_{n}$. (We consider only the indices $i$ for which $I_{n, i}^{A} \neq \varnothing$.)

- $Y_{n}^{A}$ the $\mathscr{A}_{n}^{A}$-measurable function defined by $Y_{n}^{A}(t)=T\left(\chi_{I_{n, i}^{A}} / m\left(I_{n, i}^{A}\right)\right)$, if $t \in I_{n, i}^{A}$, where $T$ is the operator canonically associated to $\left(Y_{n}, \mathscr{A}_{n}\right)_{n \in \mathbf{N}}$.

It is clear that $\left(Y_{n}^{A}, \mathscr{A}_{n}^{A}\right)_{n \in \mathbf{N}}$ is an $A$-supported martingale which satisfies

$$
Y_{n}^{A}(t) \in \bar{S}\left(C, f_{n}(t), \alpha_{n}(t)\right) \text { for every } n \in \mathbf{N}, t \in A .
$$


This is an immediate consequence of the following observation. If $B \subseteq I_{n, i}$, then $T\left(\chi_{B} / m(B)\right) \in \bar{S}\left(C, f_{n, i}, \alpha_{n, i}\right)$ where $f_{n, i}\left(\right.$ resp. $\left.\alpha_{n, i}\right)$ is the value taken by $f_{n}\left(\right.$ resp. $\left.\alpha_{n}\right)$ on the atom $I_{n, i}$ of $\mathscr{A}_{n}$.

Proof of Theorem 9. We first treat the case when $E$ is separable. Let $A$ be a measurable subset of $[0,1], m(A)>0$, and let $e \in \operatorname{ext}\left(T\left(\mathscr{F}_{A}\right)\right)^{\sim}$. To show that $e \in E_{S}$, it is sufficient to show that $\|e \wedge u\|<1 / n$ for every $n \in \mathbf{N}$.

We assume that this is not the case and show that this leads to a contradiction. Let $e_{0} \in E, e_{S} \in E_{S}$ such that $e=e_{0}+e_{S}$. Then $e \wedge u=\left(e_{0}+e_{S}\right) \wedge u=e_{0} \wedge u$, as $e_{S} \perp u$. Hence by assumption $\left\|e_{0} \wedge u\right\| \geq 1 / n_{0}$. Let now $\left(y_{\gamma}\right)_{\gamma \in \Gamma} \subseteq E_{+}$be a net such that $e_{S}=\omega^{*}-\lim _{\gamma} y_{\gamma}$. Then $\left(x_{\gamma}\right)_{\gamma \in \Gamma}=\left(y_{\gamma}+e_{0}\right)_{\gamma \in \Gamma}$ is a net in $E_{+}$ such that $e=\omega^{*}-\lim _{\gamma} x_{\gamma}$. Moreover

$$
\left\|x_{\gamma} \wedge u\right\|=\left\|\left(e_{0}+y_{\gamma}\right) \wedge u\right\| \geq\left\|e_{0} \wedge u\right\| \geq 1 / n_{0}
$$

for every $\gamma \in \Gamma$, which means that $x_{\gamma} \in H_{n_{0}}$ for every $\gamma \in \Gamma$. By Lemma 2 of [2] there exists $m \in \mathbf{N}$ such that $\overline{\mathrm{co}} H_{n_{0}} \subseteq H_{m}$. For $i \leq q(m)$ let

$$
C_{m, i}^{A}=\overline{T\left(\mathscr{F}_{I_{m, i}^{A}}\right)} .
$$

As $\left(Y_{n}^{A}, \mathscr{A}_{n}^{A}\right)_{n \in \mathrm{N}}$ is a martingale, we have that $\overline{T\left(\mathscr{F}_{A}\right)}=\overline{\operatorname{co}}\left(\bigcup_{i \leq q(m)} C_{m, i}^{A}\right)$, hence by Lemma 3 of [2], $\overline{T\left(\mathscr{F}_{A}\right)}=\operatorname{co}\left(\bigcup_{i \leq q(m)} C_{m, i}^{A}\right)$. So there exists $i_{0} \leq q(m)$ such that $e \in\left(C_{m, i_{0}}^{A}\right)^{\sim}$.

On the other hand, as $\left(Y_{n}^{A}, \mathscr{A}_{n}^{A}\right)_{n \in \mathbf{N}}$ satisfies property $(*)$, we deduce that $C_{m, i_{0}}^{A} \cap H_{m}=\varnothing$, hence also $C_{m, i_{0}}^{A} \cap \overline{\mathrm{co}} H_{n_{0}}=\varnothing$. By the Hahn-Banach theorem there exist $\beta<1$ and $\varphi \in E^{*}$ such that $1<\inf _{x \in C_{m . i_{0}}^{A}}\langle\varphi, x\rangle$ and $\sup _{x \in H_{n_{0}}}\langle\varphi, x\rangle<\beta$. Hence we also have that $\left\langle\varphi, x_{y}\right\rangle<\beta<1$ for every $\gamma \in \Gamma$, as $\left(x_{\gamma}\right)_{\gamma \in \Gamma} \subseteq H_{n_{0}}$. But this contradicts the fact that $e=\omega^{*}-\lim _{\gamma} x_{\gamma} \in\left(C_{m, i_{0}}^{A}\right)^{\sim}$.

This concludes the proof of Theorem 9 in the separable case.

If $E$ is a nonseparable Banach lattice not containing $c_{0}$ and failing RNP, then there exists a separable sublattice $F$ of $E$, which can be supposed to be a band in $E$, also failing RNP and not containing $c_{0}$. To prove Theorem 9 in the nonseparable case it is clear that it is sufficient to prove the following

Claim. $i^{* *}\left(F_{S}\right) \subseteq E_{S}$, where $i: F \rightarrow E$ is the canonical injection of $F$ into E.

Proof of the claim. Let $\pi: E \rightarrow E$ be the band projection on $F$. Then $\pi^{* *}$ : $E^{* *} \rightarrow E^{* *}$ is also a band projection. Let $P: E^{* *} \rightarrow E^{* *}$ be the band projection on $E$. Then

$$
P\left(F^{00}\right)=P\left(\pi^{* *}\left(E^{* *}\right)\right)=\pi^{* *}\left(P\left(E^{* *}\right)\right)=\pi^{* *}(E)=F,
$$

since $\pi^{* *}\left(E^{* *}\right)=F^{00}$ and since the band projections commute. This implies 
that $F^{00}=i(F) \oplus\left(F^{00} \cap E_{S}\right)$. On the other hand $F^{00}=i^{* *}\left(F^{* *}\right)=i(F) \oplus$ $i^{* *}\left(F_{S}\right)$. It follows that $i^{* *}\left(F_{S}\right)=F^{00} \cap E_{S}$, since $i^{* *}$ is a lattice isomorphism between $F^{* *}$ and $F^{00}$. The claim is then proved.

Proposition 10. Let $E$ be a Banach lattice failing RNP and not containing $c_{0}$. Then for every $\varepsilon>0$ there exists a closed convex set $C \subseteq B(E)_{+}$such that $\operatorname{ext}(\widetilde{C}) \subseteq E_{S}$ and such that $\operatorname{dist}[\operatorname{ext}(\widetilde{C}), E]>1-\varepsilon$.

Proof. Notice that it is sufficient to show the result in the separable case. Indeed, if $E$ is nonseparable, we choose a separable band $F$ in $E$ also failing RNP and not containing $c_{0}$. Hence, for $\varepsilon>0$ given, there exists a closed convex set $C \subseteq B(F)_{+}\left(\subseteq B\left(E_{+}\right)\right)$such that $\operatorname{dist}[\operatorname{ext}(\widetilde{C}), F]>1-\varepsilon$ and such that $\operatorname{ext}(\widetilde{C}) \subseteq F_{S}$. Since $F_{S} \subseteq E_{S}$ and since $\operatorname{dist}[e, F]=\|e\|=\operatorname{dist}[e, E]$ for every $e \in F_{S}$, the result also holds in the nonseparable case.

Let now $E$ be a separable Banach lattice failing RNP and not containing $c_{0}$. Let $T$ be the operator given by Theorem 9 and let $T^{* *}: L^{1 * *} \rightarrow E^{* *}$ be its second adjoint. We identify $L^{1 * *}$ with $M(K)$, the space of Radon measures on $K$, where $K$ is the spectrum of $L^{\infty} . \hat{m}$ denotes the measure on $K$ corresponding to the Lebesgue measure $m$ on $[0,1]$. As in [9], for every $\omega \in K$ we put $\varphi(\omega)=\operatorname{dist}\left[T^{* *} \varepsilon_{\omega}, E\right]$, where $\varepsilon_{\omega}$ denotes the Dirac measure at $\omega$. By [9, Lemma 7.1], we have that $\|\varphi\|_{L^{\infty}(K, \hat{m})}=\delta>0$, as $T$ is not representable. Hence for $\varepsilon>0$ given, there exists $A \subseteq[0,1], m(A)>0$ such that $\varphi(\omega)>\delta-\varepsilon$ for every $\omega \in \widehat{A}$, where $\widehat{A}=\left\{s \in K:\left\langle s, \mathbf{1}_{A}\right\rangle=1\right\}$, (follows from [12], 1.4.3a). We put $C_{0}=\overline{T\left(\mathscr{F}_{A}\right)}$. Then $\widetilde{C}_{0}=\left\{T^{* *} \mu: \mu \in M(\widehat{A})\right.$, $\|\mu\| \leq 1, \mu \geq 0\}$, and $\operatorname{ext}\left(\widetilde{C}_{0}\right) \subseteq\left\{T^{* *} \varepsilon_{\omega}: \omega \in \widehat{A}\right\}$. Hence every $e \in \operatorname{ext}\left(\widetilde{C}_{0}\right)$ satisfies $\operatorname{dist}[e, E]>\delta-\varepsilon$. On the other hand $\operatorname{ext}\left(\widetilde{C}_{0}\right) \subseteq E_{S}$ by Theorem 9 , which by the Krein-Milman theorem implies that $\widetilde{C}_{0} \subseteq \delta B\left(E^{* *}\right)$, since every $e \in E_{S}$ satisfies $\|e\|=\operatorname{dist}[e, E] \leq \delta$. If we put $C=\frac{1}{\delta} C_{0}, C$ satisfies the statements of Proposition 10, in the separable case.

Now we are ready for the

Proof of Theorem 2. For $\varepsilon>0$ given, let $C$ be the set given by Proposition 10 . We prove that the set $D=S(C)$ satisfies the statements of Theorem 2.

(i) If $e \in \operatorname{ext}(\widetilde{D})$, then $|e| \in \operatorname{ext}(\widetilde{C})$ (apply Lemma 4 and Corollary 6). By Proposition 10, $|e| \in E_{S}$, hence $e \in E_{S}$ and

$$
\operatorname{dist}[e, E]=\|e\|=\operatorname{dist}[|e|, E]>1-\varepsilon .
$$

(ii) First we show that all the slices of $C$ have diameter bigger than $2-4 \varepsilon$. This will imply that the same conclusion holds for $D$ (using a representation theorem).

Let $S=S\left(y^{*}, \alpha\right)$ be a slice of $C$ and let us prove that $S$ has diameter bigger than $2-4 \varepsilon$. 
Let $e \in \widetilde{S} \cap \operatorname{ext}(\widetilde{C})$. By Proposition 10 we have that $\|e\|>1-\varepsilon$, hence there exists $x^{*} \in E_{+}^{*},\left\|x^{*}\right\|=1$, such that $\left\langle e, x^{*}\right\rangle>1-2 \varepsilon$. Define a slice $T$ of $C$ by $T=S\left(x^{*}, 2 \varepsilon\right)$. Since $\widetilde{T}$ contains $e$ and since $e \in \operatorname{ext}(\widetilde{C}), \widetilde{T} \cap \widetilde{S}$ contains a $\omega^{*}$-slice $R$ of $\widetilde{C}$, such that $e \in R$ (see for example [3], Proposition 25.13).

Let $x \in R \cap C$. Then $e \wedge x=0$ since $e \in E_{S}$. This implies that $|e-x|=$ $|e+x|$ and consequently

$$
\|e-x\|=\|e+x\| \geq\left\langle e+x, x^{*}\right\rangle>2-4 \varepsilon .
$$

Hence every slice of $C$ has diameter bigger than $2-4 \varepsilon$.

We will see now that this also holds for $D=S(C)$. Notice first that without loss of generality, we can suppose that $E$ is separable. Then, as $E \not \supset c_{0}, E$ and $E^{*}$ can be represented as Koethe function spaces over some measure space $(\Omega, \Sigma, \mu)[8, \mathrm{II}]$. Let $y^{*} \in E^{*}$ and let $f \in L^{\infty}(\Omega, \mu),|f| \equiv 1$ be such that $y^{*}=f \cdot\left|y^{*}\right|$. Then for every $\alpha>0$

$$
S\left(D, y^{*}, \alpha\right)=\left\{f \cdot x: x \in S\left(D,\left|y^{*}\right|, \alpha\right)\right\} \supseteq\left\{f \cdot x: x \in S\left(C,\left|y^{*}\right|, \alpha\right)\right\}
$$

since $\sup _{C}\left|y^{*}\right|=\sup _{D} y^{*}$. This implies that

$$
\operatorname{diam} S\left(D, y^{*}, \alpha\right) \geq \operatorname{diam} S\left(C,\left|y^{*}\right|, \alpha\right)>2-4 \varepsilon, \quad \text { since }|f| \equiv 1 .
$$

\section{APPLICATIONS TO RENORMINGS}

Our first renorming result is in the spirit of Theorems 1 and 2 of the preceding section. We will prove that every Banach lattice failing RNP has equivalent lattice norms whose unit balls are "bad" with optimal constants for the phenomena of both far extreme points and big slices (compare with [9]). More precisely, we have:

Theorem 11. Let $E$ be a Banach lattice failing RNP. Then for every $\varepsilon>0$ there exists an equivalent lattice norm $|\cdot|$ on $E$ such that

(i) $\operatorname{dist}_{|\cdot|}\left[\operatorname{ext}\left(B_{|\cdot|}\left(E^{* *}\right), E\right]>1-\varepsilon\right.$

(ii) for every slice $S$ of $B_{|\cdot|}(E)$ one has: $\operatorname{diam}_{|\cdot|} S>2-\varepsilon$.

Proof.

Case 1. $E$ does not contain $c_{0}$.

Let $\varepsilon>0$ be given and let $D$ be the solid, convex, closed subset of $B(E)$ given by Theorem 2. Define a new lattice norm $|\cdot|$ by $B_{|\cdot|}(E)=\overline{D+\varepsilon B(E)}$. As in [9] it can be shown that this norm satisfies the statements of the theorem.

Case 2. E contains $c_{0}$.

We will also follow the methods used in [9]. The proof is similar to the one above. Let $\varepsilon>0$ be given and let $D$ be the solid, convex, closed subset of $B(E)$ given by Theorem 1 . Define first a new lattice norm $\llbracket \cdot \rrbracket$ by $B_{[\cdot]}(E)=$ $\frac{\varepsilon}{2(1-2 \varepsilon)} B(E)$. Again as in [9] it can be shown that for this norm the set $D$ satisfies

$$
\operatorname{dist}_{[\cdot]}[\operatorname{ext}(\widetilde{D}), E]>1-2 \varepsilon \text {. }
$$


Moreover, it is easily seen that all the slices of $D$ have $\mathbb{I} \cdot \mathbb{\eta}$-diameter bigger than $2-2 \varepsilon$. Similarly to case 1 , the lattice norm defined by $B_{i \cdot \mid}(E)=\overline{D+\varepsilon B_{[\cdot]}(E)}$ satisfies the statements of the theorem.

Our next renorming result deals with rough norms. Let us briefly recall the connection between roughness properties and differentiability properties in Banach spaces. It is well known that every norm on an Asplund space is Fréchetdifferentiable at some point. On the other hand it is also known ([5], [7]) that every non-Asplund space has an equivalent norm which is uniformly nonFréchet-differentiable (the uniformity constant lies in ]0,1[). It is still open whether such a rough norm can be constructed with uniformity constant almost 1 (which is the optimal value). However the answer is positive for Banach spaces containing $l^{1}$. This follows from a result of Godefroy/Maurey [5] (separable case) and Godefroy [6] (nonseparable case). In particular, the answer is positive for non-Asplund Banach lattices (as they contain $l^{1}$ ).

In this case it is natural to ask whether such a norm can be chosen to be a lattice norm. Our next theorem shows that this is indeed possible.

Theorem 12. Let $E$ be a non-Asplund Banach lattice. Then for every $\varepsilon>0$ there exists an equivalent lattice norm $|\cdot|$ on $E$ such that for every $x \in E$, $|x|=1$, there exists $y \in E,|y|=1$ such that $|x+\lambda y| \geq(1-\varepsilon)[1+|\lambda|]$ for every $\lambda \in \mathbf{R}$.

Proof. Let $\varepsilon>0$ be given and let $D \subseteq B\left(E^{*}\right)$ be the solid closed convex set given by Theorem 1, resp. Theorem 2. By Lemma $5, \bar{D}^{*}$ is again solid. Hence $\bar{D}^{*}+\varepsilon B\left(E^{*}\right)$ is the unit ball of an equivalent dual lattice norm on $E^{*}$, whose predual norm on $E$ will be denoted by $|\cdot|$. This new norm is such that all the $\omega^{*}$-slices of $B_{|\cdot|}\left(E^{*}\right)$ have $|\cdot|$-diameter bigger than $2-3 \varepsilon$. (Use the same techniques as in [9] and the fact that $S\left(\bar{D}^{*}, x, \alpha\right) \supseteq S(D, x, \alpha)$ for $x \in E$.)

We prove that the norm $|\cdot|$ satisfies the statement of the theorem. Let $x \in E,|x|=1$. Since $S=S\left(B_{|\cdot|}\left(E^{*}\right), x, \varepsilon\right)$ has $|\cdot|$-diameter bigger than $2-3 \varepsilon$, choose $x_{1}^{*}, x_{1}^{*} \in S, y \in E,|y|=1$, such that $\left\langle x_{1}^{*}-x_{2}^{*}, y\right\rangle>2-4 \varepsilon$. Hence $\left\langle x_{1}^{*}, y\right\rangle>1-4 \varepsilon$ and $-\left\langle x_{2}^{*}, y\right\rangle>1-4 \varepsilon$. Let now $0 \leq \lambda \in \mathbf{R}$. Then

$$
|x+\lambda y| \geq\left\langle x+\lambda y, x_{1}^{*}\right\rangle \geq(1-4 \varepsilon)[1+\lambda] .
$$

Similarly for $\lambda<0$, using $x_{2}^{*}$ we obtain $|x+\lambda y| \geq(1-4 \varepsilon)[1-\lambda]$. Since $\varepsilon$ is arbitrary, this concludes the proof of the theorem.

\section{ACKNOWLEDGMENT}

I want to thank G. Godefroy, A. Sersouri and M. Talagrand for helpful conversations. 


\section{REFERENCES}

1. J. Bourgain, A geometric characterization of the RNP in Banach spaces, Compositio Math. $\mathbf{3 6}$ 1978, 3-6.

2. J. Bourgain and M. Talagrand, Dans un espace de Banach réticulé solide, la propriété de RadonNikodým et celle de Krein-Milman sont équivalentes, Proc. Amer. Math. Soc. 81 1981, 93-96.

3. G. Choquet, Lectures on Analysis. II, W. A. Benjamin, New York, 1969.

4. J. Diestel and J. J. Uhl, Vector measures, Math. Surveys Monogr., vol. 15, Amer. Math. Soc., Providence, R. I., 1977.

5. G. Godefroy and B. Maurey, Normes lisses et anguleuses sur les espaces de Banach séparables, unpublished.

6. G. Godefroy, Metric characterization of first Baire class linear forms and octahedral norms (to appear in Studia Math.).

7. K. John and V. Zizler, On rough norms on Banach spaces, Comment. Math. Univ. Carolin. 19-2 (1978), 335-349.

8. J. Lindenstrauss and L. Tzafriri, Classical Banach spaces. I, II, Ergeb. Math. Grenzgeb. 92, 97 Springer-Verlag, Berlin and New York, 1977, 1979.

9. W. Schachermayer, A. Sersouri and E. Werner, Some geometrical results concerned with the Radon-Nikodym Property of Banach spaces, (to appear in Israel J. of Math.).

10. H. H. Schaefer, Banach lattices and positive operators, Grundlehren Math. Wiss., vol. 215, Springer-Verlag, Berlin and New York, 1974.

11. C. Stegall, The RNP in Banach spaces I, lectures given in Essen, Germany, in July 1982.

12. M. Talagrand, Pettis integral and measure theory, Mem. Amer. Math. Soc. 3071984.

Université Paris Vi, Tour 46-4ème Etage, 4, Place Jussieu, 75005-Paris France

Current address: Department of Mathematics and Statistics, Case Western Reserve University, Cleveland, Ohio 44106 